\title{
Combinación de Aprendizaje Cooperativo e Individual en una Asignatura de Química de Materiales
}

\author{
Massimo Lazzari \\ Universidad de Santiago de Compostela, Departamento de Química Física, Facultad de Química, Campus \\ Vida, 15782 Santiago de Compostela-España (e-mail: massimo.lazzari@usc.es)
}

Recibido Abr. 29, 2014; Aceptado Jun. 13, 2014; Versión final recibida Ago. 4, 2014

\begin{abstract}
Resumen
Se detalla la aplicación de distintas técnicas de aprendizaje cooperativo en una asignatura de química de materiales para estudiantes de diferentes facultades científicas. Desde el curso 2010/2011 parte de los temas del programa se desarrollaron mediante la técnica puzzle de Aronson, de juego-concurso o de grupo de investigación, además de las tradicionales clases magistrales y prácticas competitivas. La acogida de los cambios en la metodología didáctica por parte de los estudiantes ha sido positiva, tal y como ha resultado reflejado en las encuestas de evaluación del profesorado y en el incremento del porcentaje de aprobados. Por el otro lado, además de los comentarios positivos de los alumnos centrados el reconocimiento de la importancia del desarrollo de habilidades de trabajo grupal, se debe destacar algunas limitadas opiniones negativas relacionadas con la falta de costumbre al aprendizaje a través de técnicas de enseñanza no tradicionales.
\end{abstract}

Palabras clave: aprendizaje cooperativo, técnica puzzle de Aronson, aprendizaje activo

\section{Combination of Cooperative and Individual Learning Techniques in a Material Chemistry Course}

\begin{abstract}
The application of various cooperative learning techniques in a material chemistry course for students of different scientific faculties is reported. Since 2010/2011, part of the program has been developed through jigsaw classroom, learning games or team learning techniques, in addition to classical lectures and competitive practices. The reception of the changes in teaching methods by the students has been positive, as it has been reflected in the evaluation surveys of teachers and in the increasing of the passing rate. On the other hand, in addition to the positive feedback from students, mainly focused on the recognition of the importance of developing skills in group work, some few negative opinions mainly due to the lack of habit of working through nontraditional teaching techniques, must be mentioned.
\end{abstract}




\section{INTRODUCCIÓN}

El aprendizaje individual en el aula sigue siendo el tipo de interacción más difundido a todos los niveles educativos y sobre todo en las aulas universitarias. Mediante este acercamiento clásico a la enseñanza el profesor se dirige por igual a todos los alumnos y resuelve individualmente las dudas o los problemas que van surgiendo en ellos, de manera que cada alumno se centre únicamente en sus tareas para lograr resultados positivos. La consecución de los objetivos individuales no influye por lo tanto en los resultados de los demás y la recompensa viene determinada por el trabajo personal sin prever ningún tipo de interacción y sin tener en cuenta el trabajo de los compañeros de clase.

Aunque no hay que descartar a priori las metodologías de aprendizaje que se centran en el desarrollo individual, a menudo se considera que algunas de las carencias típicas de los estudiantes (centrándonos sobre todo en las aulas universitarias) como son la falta de interés durante la clase, una limitada capacitad de razonar y extraer en clase ideas clave para desarrollarlas posteriormente y una deficiente expresión oral (Tejedor y García-Valcárcel, 2007), se deban precisamente a la aplicación de metodologías tradicionales. Éstas no serían adecuadas para motivar el alumno y tampoco para fomentar la asunción de competencias transversales en su conjunto y, de manera más específica, de las competencias interpersonales (es decir de las capacidades que permiten mantener una buena relación social) y sistémicas (relacionadas con la visión de conjunto y la capacidad de gestionar adecuadamente la totalidad de la actuación docente) (Prieto, 2007).

En este sentido, lo que se intenta estimular en actividades llevadas a cabo aplicando técnicas de aprendizaje no tradicionales y sobre todo dentro de las así llamadas actividades de aprendizaje cooperativo (Johnson et al., 1993) es el desarrollo de habilidades y destrezas sociales, con claros beneficios para el aprendizaje de los alumnos, que van desde una mayor motivación hasta una más limitada incidencia de las carencias antes citadas (Goikoetxea y Pascual, 2002). A diferencia de lo que ocurre en las situaciones de aprendizaje individualista, en las de trabajo en equipo los alumnos tienen que trabajar conjuntamente y se logran los objetivos solamente si cada miembro del grupo consigue los suyos. Más concretamente, el aprendizaje cooperativo es un método que abarca un conjunto de técnicas que se basan en el uso didáctico de "equipos reducidos de alumnos, generalmente de composición heterogénea en rendimiento y capacidad, aunque ocasionalmente pueden ser más homogéneos, utilizando una estructura de la actividad tal que asegure al máximo la participación igualitaria (para que todos los miembros del equipo tengan las mismas oportunidades de participar) y se potencie al máximo la interacción simultánea entre ellos, con la finalidad de que todos los miembros de un equipo aprendan los contenidos escolares, cada uno hasta el máximo de sus posibilidades y aprendan, además, a trabajar en equipo" (Pujolas, 2009). De manera muy esquemática, combinando las propuestas de los autores más influyentes en el desarrollo tanto teórico como practico del aprendizaje cooperativo (Johnson et al., 1993; Kagan, 1994), los elementos básicos que estructuran estas actividades son: i) la interdependencia positiva entre los miembros del equipo; ii) la interacción simultánea entre ellos, con el objetivo de que todos los miembros de un equipo aprendan los contenidos académicos, cada uno hasta el máximo de sus posibilidades; iii) la responsabilidad individual de cada miembro de conseguir las metas que se le han asignado; iv) participación igualitaria.

Las aplicaciones didácticas en el ámbito universitario de las técnicas de aprendizaje cooperativo en disciplinas científicas e ingenierías son bastante numerosas (Felder y Brent, 2007) y una revisión exhaustiva iría más allá de los objetivos específicos de este artículo, donde el interés se limitará a antecedentes directamente relacionados con aplicaciones a la enseñanza de asignaturas de química. A manera de ejemplo de la difusión de aplicaciones en clases o prácticas de laboratorio, una búsqueda que incluya los términos química (o chemistry) y aprendizaje cooperativo (o cooperative learning) en el Web of Science y en el SciELO citation index permite encontrar cerca de 200 artículos publicados entre 1994 y 2013, en su mayoría en el Journal of Chemical Education. Cabe destacar que la mayor parte de ellos discuten la aplicación de situaciones de aprendizaje cooperativo a asignaturas de química física, química orgánica y ciencia de los materiales, limitando la implementación y la valoración del rendimiento a aspectos muy concretos y limitados del programa, con el desarrollo de actividades de limitada duración, a menudo módulos auto-concluyentes de una hora (Jones et al., 2012). Entre los últimos ejemplos publicados, en Karacop y Doymus (2013) se valora la efectividad del uso de una técnica de aprendizaje cooperativo como la técnica puzzle de Aronson respecto a métodos tradicionales para explicar los distintos tipos de enlace químico. La comparación entre las notas de examen obtenidas por estudiantes asignados al azar a grupos de enseñanza tradicional, de aprendizaje basado en el uso de técnicas de animación gráfica y de aprendizaje cooperativo puso en evidencia la menor capacidad de comprensión de los estudiantes sometidos a situaciones de aprendizaje individual. De manera similar, Tien et al. (2002) y Wamser (2006) demostraron que la aplicación de otra situación cooperativa como la de la técnica de grupos de investigación a la enseñanza de la química orgánica básica permite mejorar de manera considerable los resultados de los exámenes de fin de curso de los estudiantes de primer año de dos universidades de Estados Unidos. Los resultados de otra experiencia docente llevada a cabo en una universidad española 
incorporando metodologías activas basadas en la utilización del aprendizaje cooperativo, evaluación continua y las nuevas tecnologías en la enseñanza de una asignatura de Materiales de Construcción han evidenciado un aumento notable del número de aprobados respecto a los cursos anteriores, en los que se aplicaban métodos individuales (Reyes y Gálvez, 2010). Finalmente, se citan también los recién publicados trabajos de Madrid et al. (2013) y Regalado-Méndez et al. (2014).

Teniendo en cuenta estos prometedores resultados, en este artículo se describe la aplicación de distintas técnicas de aprendizaje cooperativo, además de clases magistrales y prácticas realizadas con metodologías tradicionales, en una materia de libre elección relacionada con la química de los materiales poliméricos, Degradación, Estabilización y Reciclaje de Plásticos, con el objetivo último de conseguir una mejora de la eficacia de la enseñanza. La asignatura, de 4,5 créditos (3,5 teóricos y 1 práctico), puede ser cursada por estudiantes de la Universidad de Santiago de Compostela de las facultades de Química, Física, Farmacia e Ingeniería Química, de diferentes años de carrera, siendo 20 el número máximo de plazas establecido. La aplicación de técnicas de aprendizaje no tradicionales (Johnson et al., 1993; Slavin, 1995; Thousand, 1994) tiene los siguientes objetivos parciales, sobre todo teniendo en cuenta la participación de estudiantes con maduración heterogénea y currícula académicos diferentes: i) conseguir una implicación más activa de todos los estudiantes, sobre todo en el laboratorio; ii) involucrarles activamente en su proceso de aprendizaje, con una mayor atención hacia los conocimientos con un mayor carácter aplicado; y iii) facilitar y fomentar la colaboración entre estudiantes de edad y áreas científicas distintas.

\section{TECNICAS UTILIZADAS}

Se aplican las técnicas de puzzle de Aronson (Aronson y Bridgeman 1979), de juego-concurso (DeVries y Edwards, 1973) y la técnica de grupos de investigación (Sharan, 1980), además de una práctica competitiva de laboratorio, ya que cada una de esta herramientas se adapta mejor a las necesidades didácticas de temas específicos de la asignatura. En la técnica de puzzle de Aronson (Aronson y Bridgeman 1979) se dividen los alumnos en equipos heterogéneos de 4 ó 5 miembros y a cada uno de sus componentes se le asigna y se le hace responsable de una parte diferente de la tarea a llevar a cabo. Con esta estructura de trabajo se podría decir que cada alumno posee una pieza vital de la gran totalidad, recordando por lo tanto un rompecabezas. En síntesis esta técnica consiste en los siguientes pasos: i) se organizan los equipos (grupos puzzle) para trabajar un material académico que ha sido dividido en tantos subtemas como miembros tenga el equipo; ii) cada miembro se ocupará de estudiar o aprender un subtema; iii) los diferentes miembros de los distintos equipos puzzle que van a ocuparse de las mismas tareas se reúnen en grupos de expertos para estudiarlas y discutirlas; iv) los alumnos vuelven a sus grupos puzzle y "enseñan" su sección a los compañeros. Queda evidente que esta metodología es especialmente útil para las áreas de conocimiento en las que los contenidos son susceptibles de ser "fragmentados" en diferentes partes, como son algunos temas de ciencias experimentales. En relación a los ejemplos de aplicaciones en química, se citan los trabajos de Jones et al. (2012) y Karacop y Doymus (2013).

La principal diferencia entre la técnica de grupos de investigación propuesta por Sharan (1980) y la de puzzle de Aronson consiste en permitir que los alumnos creen los grupos de trabajo, guiándose por los temas en los que están mejor preparados o que más les interesan. El número de ejemplos de aplicación en el ámbito de las ciencias experimentales es más limitado, quizás debido a esta mayor libertad de elección que requiere haber trabajado previamente habilidades sociales y de comunicación entre los alumnos. Se considera más apropiada para estudiantes de ciencias sociales. El experimento más importante en este sentido fue llevado a cabo desarrollando temas de historia y geografía (Sharan y Shachar, 1988).

La técnica de juego-concurso de DeVries, conocida también como torneos de aprendizaje (DeVries y Edwards, 1973) combina elementos competitivos con aspectos típicos del aprendizaje cooperativo, ya que en un momento de su desarrollo, grupos heterogéneos compiten entre ellos. Los alumnos siguen una secuencia de aprendizaje en la cual primero reciben el material académico, después hacen ejercicios y prácticas en grupo para asegurarse que todos los miembros se saben bien la lección. Cuando los compañeros determinan que todos están preparados, se hace finalmente la evaluación, en forma de torneo académico en que los estudiantes de cada equipo compiten con los miembros de similares niveles de rendimiento de los otros equipos para ganar puntos para sus respectivos equipos. Esta situación didáctica ofrece la ventaja de poder trabajar contenidos de materias de una forma divertida a la vez que aprenden a mejorar sus relaciones interpersonales, a integrarse mejor en el grupo, a reconocer y valorar los esfuerzos realizados por cada uno de los miembros.

\section{DESCRIPCIÓN GENERAL DE LA PROPUESTA}

Los objetivos académicos del curso, que se ha impartido durante algunos años mediante metodologías individualistas, no han sido modificados con la introducción de las técnicas de aprendizaje cooperativo, ya 
que éstas solo van a tener la función de mejorar la eficacia de la enseñanza (aunque desplazando el centro de gravedad del profesor al alumno). Por el contrario, la metodología de enseñanza (Tabla 1) ha sido modificada introduciendo distintas técnicas de aprendizaje cooperativo, tanto para el desarrollo de la parte teórica del curso como para la parte practica. Para tener en cuenta la introducción de las técnicas de aprendizaje cooperativo en los temas 1, 5, 6 y 9, y en las prácticas se han llevado a cabo algunos pequeños cambios del programa a impartir (Tabla 2).

Tabla 1: metodología de la asignatura Degradación, Estabilización y Reciclaje de Plásticos

Metodología de la enseñanza

Clases magistrales (3 horas a la semana) y aprendizaje cooperativo (técnica puzzle de Aronson, técnica juegoconcurso y técnica grupos de investigación) utilizado para el desarrollo de algunos temas específicos.

Laboratorio de técnicas básicas de análisis de materiales poliméricos (prácticas competitivas) y laboratorio de estudio de degradación (prácticas tradicionales).

Seminarios prácticos (interpretación de problemas prácticos de degradación). Seminarios impartidos por expertos y visitas a centros relacionados con los objetivos del curso

Tabla 2: contenido de la asignatura. Los temas/parte de temas a realizar a través de técnicas de aprendizaje no tradicionales se evidencian en cursiva.

Programa teórico (35 horas) + Programa practico (10 horas)

Tema 1: Edad del plástico. Polímeros en la vida cotidiana. Estabilidad y técnicas experimentales.

Tema 2: Degradación térmica.

Tema 3: Degradación termooxidativa.

Tema 4: Fotodegradación oxidativa.

Tema 5: Otros tipos de degradación. Degradación de polímeros sometidos a esfuerzos mecánicos. Biodegradación. Polímeros biodegradables en medicina y degradación in vivo.

Tema 6: Estabilización. Necesidad de la estabilización. Fotoestabilizantes y otros estabilizantes.

Tema 7: Aspectos prácticos de la degradación de plásticos.

Tema 8: Inflamabilidad. Combustión de los plásticos y retardantes de llama.

Tema 9: Plásticos y medio ambiente. Reciclaje primario y secundario (r. mecánico). Reciclaje terciario (r. químico). Recuperación de energía. Diseño industrial en función del reciclaje.

Prácticas de laboratorio: aprendizaje de las técnicas de caracterización de materiales plásticos.

\section{APLICACIÓN DEL APRENDIZAJE COOPERATIVO}

Tema 1. Después de una introducción mediante clases magistrales, la última parte del tema, estabilidad y técnicas experimentales, se lleva a cabo a través de la técnica puzzle de Aronson. Todo esto con el doble objetivo de facilitar el acercamiento entre los alumnos y permitir una mejor comprensión de una parte de programa que se podría considerar como poco estimulante si se presentara con técnicas tradicionales de enseñanza (Traver y García, 2006). Teniendo en cuenta que normalmente el número de estudiantes es de 20 , el profesor propone la formación de 5 grupos puzzle de 4 estudiantes, descomponiendo por lo tanto el trabajo en 5 partes: técnicas de análisis térmico, técnicas espectroscópicas, medidas mecánicas, técnicas de microscopia y micro-análisis. Los grupos se forman ajustándose al máximo criterio de heterogeneidad; es decir, en cada grupo entran sujetos con niveles de rendimiento diferentes, diferente año de carrera, diferentes carreras y sexo diferente. Todo el material estará disponible en el aula, donde los grupos de expertos completarán su parte en 3 horas, antes de constituir de nuevo los grupos originarios o grupos puzzle. Cada experto informa a los compañeros de grupo en un tiempo de 20-30 minutos, de forma que la última parte del trabajo lleve 2 horas, además de otras 2 horas para la redacción de un dossier por parte de cada grupo, resultando un total de 7 horas para toda esta primera actividad de cooperación. La puntuación es idéntica para cada miembro del grupo, ya que la evaluación individual de los conocimientos adquiridos se lleva a cabo dentro de la evaluación final sobre todos los temas teóricos.

Tema 5. Todo el tema se lleva a cabo a través de la técnica puzzle de Aronson, aunque variando el número de grupos y, en principio, su composición. Se constituyen 4 grupos puzzle de 5 estudiantes para tratar las siguientes partes: degradación mecánica, biodegradación y biodegradabilidad, degradación útil y degradación in vivo. En la medida de lo posible se intentará mantener la necesaria heterogeneidad en la 
formación de los grupos, intentando que no vuelvan a estar juntos los estudiantes de los grupos formados dentro de la actividad relacionada al tema 1. Solo parte del material para llevar a cabo la actividad de los grupos de expertos está disponible en el aula, ya que los estudiantes reciben información sobre dónde hacer búsquedas específicas de bibliografía a través recursos disponibles en red (biblioteca de la Facultad de Química). Se consideran necesarias 4 horas totales para terminar el trabajo de los grupos de expertos y 2 horas para la exposición de cada uno de los expertos a los compañeros de grupo, además de otras 3 horas para la redacción de un dossier por parte de cada grupo, sumando un total de 9 horas para esta segunda actividad de cooperación. La puntuación es idéntica para cada miembro del grupo.

Tema 6. El tema Estabilización y estabilizantes se adapta perfectamente a las dinámicas de la técnica juego-concurso. Se constituyen 5 grupos de 4 estudiantes, cuya heterogeneidad ya puede tener en cuenta los resultados adquiridos en las anteriores actividades de cooperación. Después de la explicación del tema por parte del profesor, los grupos tienen 2 horas para preparar las 4 partes/sujetos en las que está dividido y sobre las que tienen que concursar. En las 2 horas de clase siguientes se realizan 4 concursos de media hora cada uno, en los que participarán un miembro por grupo, de rendimiento similares. La puntuación de cada grupo, y de cada miembro del grupo, es la suma de las puntuaciones obtenidas por cada uno de los participantes de cada grupo en los 4 concursos.

Tema 9. Después de una introducción por parte del profesor, casi todo el tema Plásticos y medio ambiente es desarrollado por los estudiantes a través de proyectos de Grupos de investigación. Los estudiantes se dividen al principio del curso en 4 grupos de 5 personas y eligen un subtema de trabajo dentro de las opciones: reciclaje primario y secundario (r. mecánico), reciclaje terciario (r. químico), recuperación de energía y diseño industrial en función del reciclaje. Los estudiantes reciben unas primeras directrices sobre dónde y cómo localizar las fuentes de información y son monitorizados por el profesor durante todo el semestre, con el objetivo final de preparar un informe. La puntuación de cada grupo, y de cada miembro del grupo, depende de la evaluación, por parte de los estudiantes de los otros grupos, del informe y de la presentación final de todo el material preparado. La presentación final de los 4 grupos tiene lugar después de la presentación de la introducción del tema 9 por parte del profesor, representando las presentaciones de los 4 subtemas las últimas 2 horas de clase de toda la asignatura. Los miembros de cada grupo pueden decidir si uno o más miembros del grupo, aunque no todos, merecen una evaluación mejor que la del grupo en su totalidad.

Prácticas competitivas de caracterización e identificación de materiales plásticos. En un tiempo límite de 10 horas los 10 grupos formados teniendo en cuenta los resultados obtenidos en las anteriores actividades de cooperación, acoplando 1 estudiante con buenos resultados a uno con resultados peores, tienen que identificar un material plástico de composición desconocida. Las técnicas disponibles para la identificación son las mismas para todos los grupos, que pueden pedir consejos al profesor sobre la realización de los análisis, pero no sobre la interpretación de los mismos. Todos los grupos que acierten la identificación reciben la puntuación máxima, mientras los demás son evaluados de forma proporcional. La puntuación de los dos miembros del grupo es la misma.

\section{PROCEDIMIENTO DE EVALUACIÓN}

La evaluación de los estudiantes tiene en cuenta tanto los resultados obtenidos en cada una de las cinco experiencias de aprendizaje cooperativo como la puntuación obtenida en el examen escrito individual, que se lleva a cabo sobre los 9 temas tratados durante la asignatura, independientemente de la metodología de enseñanza. La nota final es la suma de las puntuaciones en Tabla 3. Se tienen por lo tanto en cuenta las notas de las actividades cooperativas (notas de grupo) y la nota individual del examen escrito final, promediando las capacidades individuales con la capacidad de llevar a cabo actividades colaborativas.

Tabla 3: peso de las diferentes actividades evaluadas sobre la nota final.

\begin{tabular}{|l|c|}
\hline Actividad & Peso (\%) \\
\hline Puzzle, Tema 1 & 10 \\
\hline Puzzle, Tema 5 & 10 \\
\hline Juego-concurso, Tema 6 & 10 \\
\hline Grupos investigación, Tema 9 & 20 \\
\hline Prácticas competitivas & 10 \\
\hline Examen escrito final & 40 \\
\hline
\end{tabular}




\section{APLICACIONES Y DISCUSIÓN}

Con el fin de evaluar la variación de la eficacia de la enseñanza con la aplicación a partir del curso 2010/2011 de los diferentes métodos de aprendizaje cooperativo descritos anteriormente, se hizo referencia tanto a parámetros analíticos como a las opiniones y comentarios recogidos directamente de los alumnos. En particular, en términos de acogida del método, se consideran especialmente relevantes los resultados de las encuestas de satisfacción del alumnado con la docencia, realizadas manteniendo el mismo cuestionario estándar de 7 preguntas puesto a punto por el Vicerrectorado de Calidad y Mejora de Procedimientos de la Universidad de Santiago de Compostela y utilizado desde la implantación de la materia. No se ha considerado conveniente cambiarlo para asegurar homogeneidad y continuidad en los procesos de evaluación. En la Tabla 4 se recogen las medias de las notas recibidas por el profesor en los tres últimos cursos realizados aplicando metodologías de aprendizaje individual (de 2007/2008 a 2009/2010), comparadas con los resultados del único curso posterior al cambio metodológico por el que se haya realizado dicha encuesta oficial (2011/2012). En el cuestionario se pide a los alumnos una valoración de 1 a 5 ( $1=$ nada, $2=$ poco, $3=$ =aceptable, $4=$ bastante, $5=$ mucho). El porcentaje de participantes en las encuestas ha sido constante y alrededor del $30 \%$ de media.

Tabla 4: resultados de las encuestas de evaluación del profesorado de la asignatura.

\begin{tabular}{|l|c|c|}
\hline \multirow{2}{*}{ Pregunta realizada } & \multicolumn{2}{|c|}{ Nota } \\
\cline { 2 - 3 } & $2007-2010$ & $2011 / 2012$ \\
\hline 1 El trabajo que hace en clase el/la profesor/a me ayuda a comprender la materia & 4.15 & 5 \\
\hline 2 El/la profesor/la estimula el trabajo personal de los/las estudiantes en la materia & 4.07 & 5 \\
\hline $\begin{array}{l}\text { 3 El/la profesor/a resuelve con claridad las dudas que le formulan los/las estudiantes } \\
\text { sobre la materia }\end{array}$ & 4.28 \\
\hline 4 El/la profesor/a procura conocer si los/las estudiantes entienden el que se les explica & 4.35 & 5 \\
\hline 5 Estoy satisfecho/a con la atención recibida de este/a profesor/a en el horario de tutorías & 4.50 & 4.50 \\
\hline 6 Considero que este/a profesor/a me ayudó a aprender & 4.36 & 5 \\
\hline 7 Globalmente estoy satisfecho/a con el/la profesor/a de esta materia & 4.50 & 5 \\
\hline
\end{tabular}

Aunque el cuestionario no haya sido diseñado para valorar materias impartidas de manera no tradicional, se considera que pueda aportar informaciones significativas también en éstas. Los resultados son en general satisfactorios, pasando de una valoración media global de 4.32 a 4.93, respecto a una nota media del profesorado de la universidad que se mantuvo constante alrededor de 3.5. Resulta especialmente relevante la evaluación de la labor de estímulo del profesor a comprender la materia (pregunta 2) pasada de 4.07 a 5 y más en general de satisfacción con el profesor (preguntas 1 y 7 , respectivamente de 4.15 y 4.50 a 5), que sin duda se relacionan con una mejora de la relación alumno-profesor y una exitosa acogida de las actividades innovadoras. En cualquier caso, más allá de la consideración por parte de los alumnos de un aprendizaje cualitativamente mejor (reflejada sobre todo en las preguntas 4 y 6), parece más significativo comparar el nivel de preparación de los alumnos a través de un seguimiento del número de aprobados, considerado también por otros autores (Felder y Brent, 2007; Reyes y Gálvez, 2010; Karacop y Doymus, 2013) como el parámetro más importante para poder sacar conclusiones cuantitativas.

Cabe destacar que por sus características de materia de libre elección, la asignatura ha tenido desde su implantación un elevado porcentaje de aprobados en primera convocatoria de examen y una limitada incidencia de alumnos no presentados, que hace difícil una comparación de resultados dentro de niveles, en cualquier caso, superiores a la media de la universidad (alrededor del $50 \%$ de aprobados en el periodo considerado). En la Tabla 5 se recoge la variación del porcentaje de aprobados en la primera convocatoria de examen sobre alumnos que participaron activamente a las propuestas didácticas. Para no falsar la estadística no se ha hecho referencia al total de alumnos matriculados, ya que siempre hay un porcentaje pequeño pero variable de estudiantes que sin participar a las clases tienen derecho a examen, tal y como previsto por los estatutos de la universidad, y suele presentarse en las siguientes convocatorias.

Tabla 5: porcentaje de aprobados en primera convocatoria sobre asistentes activos.

\begin{tabular}{|l|c|c|c|c|c|c|}
\hline & $2007 / 2008$ & $2008 / 2009$ & $2009 / 2010$ & $2010 / 2011$ & $2011 / 2012$ & $2012 / 2013$ \\
\hline Porcentaje de aprobados & 84 & 82 & 86 & 92 & 100 & 100 \\
\hline
\end{tabular}


No obstante la media de aprobados desde su implantación resulta ser muy alta (> 80\%), se puede notar como en comparación con el último año académico con metodología individualizada el porcentaje de aprobados subió de manera apenas apreciable en 2010/2011, pasando finalmente al valor máximo en los dos último curso. Estas cifras son muy buenas y sin duda esperanzadoras, pero necesitarían de más años de experimentación docente para poder confirmar de manera univoca la bondad de los cambios introducidos. No obstante, hay que tener también en cuenta los comentarios de los alumnos, o por lo menos de algunos de ellos, no siempre completamente positivos hacia las nuevas propuestas educativas.

La opinión de los alumnos ha sido recogida de manera informal, durante las clases magistrales o las actividades cooperativas, o a lo largo del periodo didáctico, directamente durante las tutorías o encuentros individuales con los alumnos para resolver dudas; todo ello sin olvidar los comentarios y opiniones expresadas libremente entre pares mientras se llevan a cabo las actividades en grupo. Entre los comentarios positivos, la mayoría, sobre las diferentes ventajas del trabajo en grupo, merece la pena destacar, entre los más comunes, los que hacen referencia a la importancia de la aportación de diferentes puntos de vista, de intercambio de informaciones, de comprensión del espíritu de grupo (este último valido también para la práctica competitiva). Al contrario los comentarios negativos se centran principalmente en un desacuerdo con las modalidades y los tiempos del trabajo cooperativo, a veces buscando una justificación en la supuesta pérdida de tiempo que estos conllevan o en la falta de costumbre. Siempre en relación a los puntos de vista no favorables a las actividades de aprendizaje cooperativo, hay que recordar que en cada curso algunos alumnos decidieron no participar a ellas y acudir directamente al examen escrito final. El porcentaje de estos alumnos ha sido en cualquier caso comparable a él detectado en los cursos anteriores.

\section{CONCLUSIONES}

En general, la incorporación de diferentes técnicas de aprendizaje cooperativo y de una practica competitiva a una materia de química de los materiales ha demostrado ser una herramienta muy útil para fomentar un aprendizaje activo sin perder de vista las ventajas de una enseñanza llevada a cabo con metodología tradicional, por lo menos en los temas que mejor se adaptan a un trabajo individual. Más específicamente, de los antecedentes de la literatura expuestos, de los argumentos y aplicaciones presentadas y de la discusión de los resultados se pueden obtener las siguientes conclusiones puntuales:

i) La aplicación de métodos activos paralelamente a la impartición de clases magistrales ha conllevado a un aumento del porcentaje de aprobados.

ii) La acogida de los cambios en la metodología didáctica por parte de los estudiantes ha sido positiva, tal y como ha resultado reflejado en las encuestas de evaluación del profesorado. La nota media ha pasado de un valor ya elevado al valor máximo en casi todos los aspectos cubiertos por la encuesta, dentro de los que cabe destacar el grado de satisfacción por la labor de estímulo del profesor a comprender la materia y, más en general, con el profesor.

iii) Aunque los comentarios informales de los alumnos hayan sido mayoritariamente favorables, existen opiniones de alumnos resistentes o incluso hostiles hacia el trabajo en grupo.

iv) La mejor solución para enfrentarse a este limitado rechazo está no solo en la mejor puesta a punto de las actividades innovadoras dentro de la materia si no sobretodo en una deseable y creciente propuesta de metodologías de aprendizaje no tradicionales en el conjunto de actividades académicas del alumnado.

\section{REFERENCIAS}

Aronson, E., y Bridgeman, D., Jigsaw groups and the desegregated classroom: In pursuit of common goals, Personality and Social Psychology Bulletin, 5, 438-446 (1979).

DeVries, D. y Edwards, K., Learning games and student teams: Their effect on the classroom process, Amer. Educ. Research J., 10, 307-318 (1973).

Felder, R.M. y Brent, R., Cooperative Learning, en Mabrouk, P.A. ed., Active Learning: Models from the Analytical Sciences, ACS Symp. Ser. 970. ACS, Washington, USA, cap. 4, 34-53 (2007)

Goikoetxea, E. y Pascual, G. G. Aprendizaje cooperativo: bases teóricas y hallazgos empíricos que explican su eficacia, Educación XX1, 5, 227-247 (2002). 
Johnson, D.W., Johnson, R.T. y Holubec, E.J., Cooperation in the Classroom (6th ed.), Interaction Book Company, Edina, USA (1993).

Jones, T.N., Graham, K. J. y Schaller, C.P., A jigsaw classroom activity for learning IR analysis in organic chemistry, J. Chem. Ed., 89, 1293-1294 (2012).

Kagan, S., Cooperative Learning, Kagan Publishing, San Clemente, USA (1994).

Karacop, A. y Doymus, K., Effects of jigsaw cooperative learning and animation techniques on students' understanding of chemical bonding and their conceptions of the particulate nature of matter. J. Sci. Educ. Technol. 22, 186-203 (2013).

Madrid J.C., Arellano M., Jara R., Merino C. y Balocchi E. El aprendizaje cooperativo en la comprensión del contenido "disoluciones". Un estudio piloto. Educación química, 24 (núm. extraord. 2), 471-479 (2013).

Prieto, L., El aprendizaje cooperativo, PPC, Madrid, España (2007).

Pujolas, P., La calidad en los equipos de aprendizaje cooperativo. Algunas consideraciones para el cálculo del grado de cooperatividad, Revista de Educación, 349, 225-239 (2009).

Regalado-Méndez A., Delgado-Vidal F.K., Martínez-López R.E. y Peralta-Reyes E. Balanceo de Ecuaciones Químicas Integrando las Asignaturas de Química General, Algebra Lineal y Computación: Un Enfoque de Aprendizaje Activo. Formación Universitaria, 7(2), 29-40 (2014).

Reyes, E. y Gálvez, J., Experiencias docentes en innovación educativa como mejora de una enseñanza tradicional de los materiales de construcción, Formación Universitaria, 3(4), 13-24 (2010).

Sharan, S., Cooperative Learning in teams: recent methods and effects on achievement attitudes and ethic relations, Review of Educ. Research, 50, 241-271 (1980).

Sharan, S. y Shachar, H, Language and learning in the cooperative learning classroom. Springer, New York, USA (1988).

Slavin, R., Cooperative Learning, Allyn \& Bacon, Massachusetts, USA (1995).

Tejedor, F.J. y García-Valcárcel, A., Causas del bajo rendimiento del estudiante universitario (en opinión de los profesores y alumnos). Propuestas de mejora en el marco del EEES, Revista de Educación, 342, 443473 (2007).

Thousand, J.S, Creativity and Collaborative Learning: A Practical Guide to Empowering Students and Teachers, Brooks Publishing Co., Baltimore, USA (1994).

Tien, L.T., Roth, V. y Kampmeier, J.A., Implementation of a Peer-Led Team Learning Approach in an Undergraduate Organic Chemistry Course, J. Research Sci. Teaching, 39, 606-632 (2002).

Traver Martí, J.A. y García López, R., La Técnica Puzzle de Aronson como Herramienta para Desarrollar la Competencia "Compromiso Ético" y la Solidaridad en la Enseñanza Universitaria, Revista Iberoamericana de Educación, 40(4), 1-9 (2006).

Wamser, C.C., Peer-Led team learning in organic chemistry: effects on student performance, success, and persistence in the course, J. Chem. Ed., 83, 1562-1566 (2006). 